\title{
Medi ouiz
}

\section{3-Jährige Frau mit schmerzhaften Rötungen an beiden Unterschenkeln}
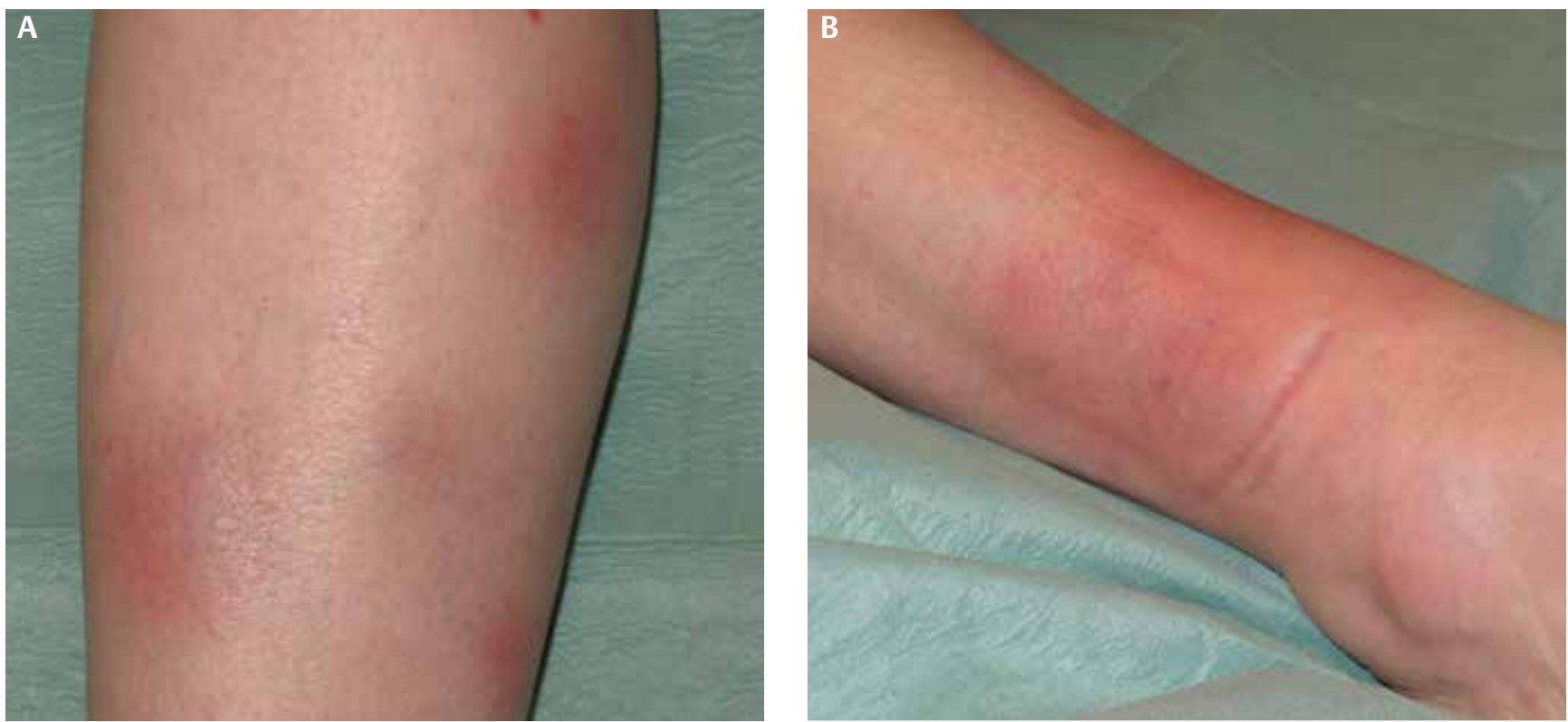

Abb. 1 Übersichtsaufnahme des rechten Unterschenkels (A), Detailaufnahme des rechten Knöchels (B).

Dr. med. Jörg Felber ${ }^{1}$

Dr. med. Marko Weber ${ }^{1}$

Dr. med. Benjamin T. Schleenvoigt ${ }^{2}$

${ }^{1}$ Klinik für Innere Medizin IV,

Universitätsklinikum Jena

${ }^{2}$ Zentrum für Infektionsmedizin und

Krankenhaushygiene, Universitäts-

klinikum Jena

Korrespondenz:

joerg.felber@med.uni-jena.de
Eine 23-jährige Frau hat seit 6 Wochen abdominelle Schmerzen und intermittierende, schmerzhafte Rötungen an beiden Unterschenkeln. Die körperliche Untersuchung ergibt eine druckschmerzhafte Resistenz im rechten Unterbauch. Bei der Inspektion der Beine zeigt sich der in $\triangleright$ Abb. 1 dargestellte pathologische Hautbefund.

- Welcher Befund ist es?

- Erlauben der Befund und die Klinik eine Verdachtsdiagnose?

- Wenn ja, welche?

- Sind Differenzialdiagnosen möglich?

- Wenn ja, welche? 


\title{
Medi \\ 23-jährige Frau mit schmerzhaften Rötungen an beiden Unterschenkeln
} ouiz

\author{
Quiz siehe S. 1581
}
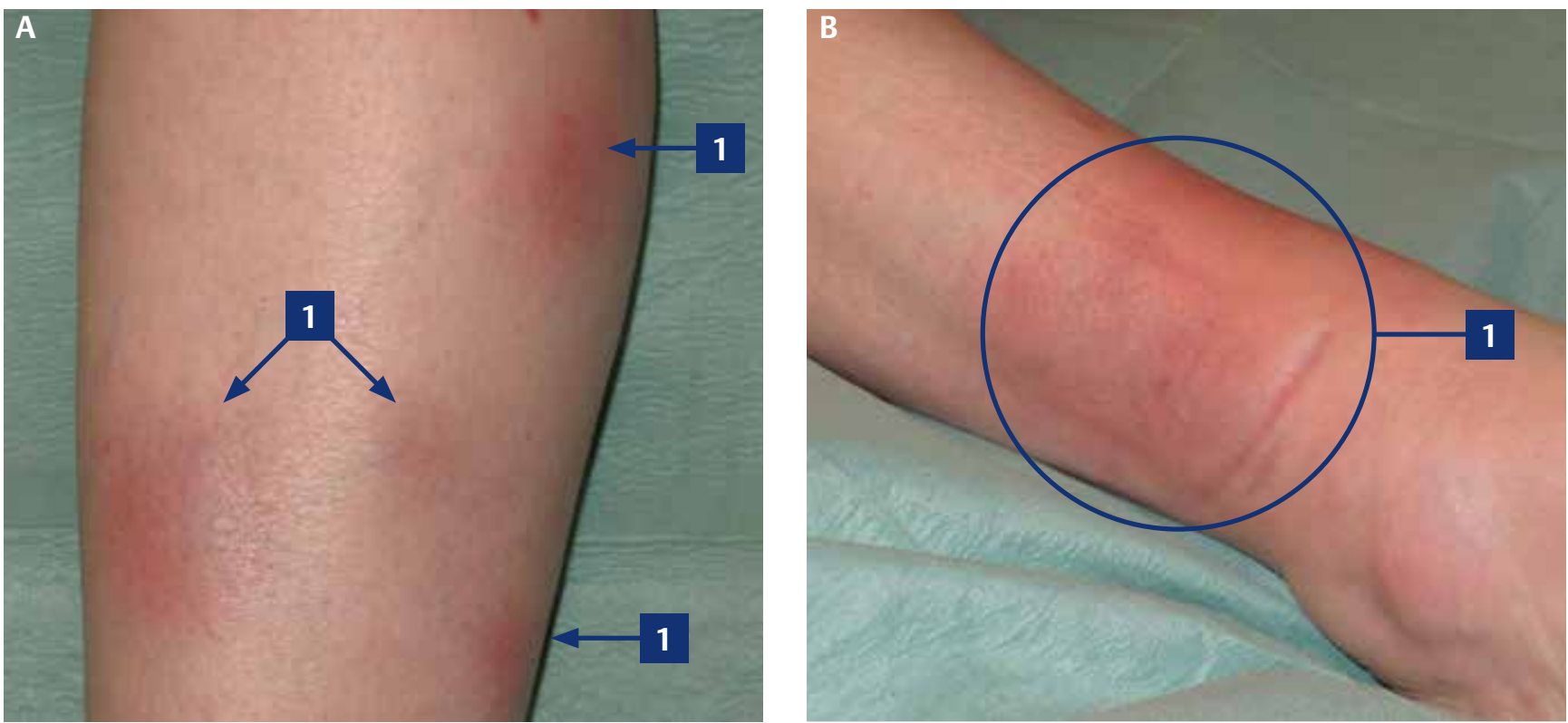

\section{Befund}

1. knötchenförmige Hautrötungen an beiden Unterschenkeln

\section{Diagnose}

- Erythema nodosum bei M. Crohn mit entzündlicher Aktivität

\section{Differenzialdiagnosen}

- Sarkoidose, Löfgren-Syndrom

- Infektionen (Tuberkulose, Streptokokken, Yersinien, Chlamydien, Katzenkratzkrankheit, Toxoplasmose)

- Reaktion auf Arzneimittel (z.B. Sulfonamide, orale Kontrazeptiva)

- rheumatisches Fieber

- Morbus Behçet

\section{Erläuterung}

Hautveränderungen sind ein häufiger Befund bei Patienten mit chronisch entzünd- lichen Darmerkrankungen (CED) [1]. Neben der Haut kann sich eine CED extraintestinal auch

- okulär (Uveitis, Episkleritis, Skleritis),

- muskuloskeletal (periphere Arthritis, axiale Arthropathien) und

- hepatobiliär (primär sklerosierende Cholangitis, chronische Autoimmunhepatitis, Overlap-Syndrom).

manifestieren [2, 3]. Das Pyoderma gangraenosum gehört ebenfalls zu den CEDassoziierten Hauterkrankungen [4].

Bei einem Erytema nodosum ist das subkutane Fettgewebe unter Beteiligung der Kapillarwände akut entzündet. In der Folge bilden sich die charakteristischen schmerzhaften Knötchen. Typische Prädilektionsstellen sind

- Unterschenkelstreckseiten,

- Kniegelenke,

- Sprunggelenke,

- seltener auch Arme und Gesäß.

Es handelt sich um eine allergische Überreaktion (Typ III) der Haut. Der Befund erlaubt eine Blickdiagnose. Das Erythem kann im Zusammenhang mit zahlreichen
Grunderkrankungen auftreten [5]. Um bei einem klinischen Verdacht die Ursache einzugrenzen, können u.a. folgende Untersuchungen sinnvoll sein:

- Stuhluntersuchung (Yersinien),

- Rachenabstrich (Streptokokken),

- Röntgen-Thorax (Sarkoidose, Tuberkulose)

- endoskopische Untersuchungen (CED)

Die Therapie besteht vor allem darin, die zugrundeliegende Erkrankung zu behandeln bzw. das auslösende Agens abzusetzen. Zur symptomatischen Therapie gehören

- nicht-steroidalen Antiphlogistika (z.B. Indometacin, Acetylsalicylsäure) und

- Kaliumjodid (lokal) [5].

Literatur

1 Isene R, Bernklev T, Høie O et al. Extraintestinal manifestations in Crohn's disease and ulcerative colitis: results from a prospective, population-based European inception cohort. Scand J Gastroenterol 2015; 50: 300-305 http://dx.doi.org/10.1055/s-0041-103199 
2 Van Assche G, Dignass A, Bokemeyer B et al. Second European evidence-based consensus on the diagnosis and management of ulcerative colitis part 3: special situations. J Crohns Colitis 2012; 7: 1-33

3 Van Assche G, Dignass A, Reinisch W et al. The second European evidence-based Consensus on the diagnosis and management of Crohn's disease: Special situations. J Crohns Colitis 2010; 4: 63-101

4 Weizman A, Huang B, Berel D et al. Clinical, serologic, and genetic factors associated with pyoderma gangrenosum and erythema nodosum in inflammatory bowel disease patients. Inflamm Bowel Dis 2014; 20: 525-533

5 Blake T, Manahan M, Rodins K. Erythema nodosum - a review of an uncommon panniculitis. Dermatol Online J 2014; 20 : 22376 